\title{
Role of 18-FDG/PET in evaluation of hepatocellular carcinoma managed locally by transarterial chemoembolization compared to triphasic computed tomography
}

Manal Ibraheim Gomaa

\begin{abstract}
Background: This study was done to evaluate the role of FDG/PET compared to CECT in evaluating HCC after local interventional treatment. We evaluate forty-six patients with known history of TACE as a locoregional treatment of pathologically proved HCC. There were forty-three males and three females.

Results: The collected data were statistically analyzed, and we found that FDG-PET has a sensitivity of 94\%, specificity of $84 \%$, positive predictive value of $86.7 \%$, negative predictive value of $86.4 \%$, and accuracy of $93.4 \%$ in the detection of residual or recurrent at the managed tumor bed compared to CECT which shows a sensitivity of $94 \%$, specificity of $84 \%$, positive predictive value of $86.7 \%$, negative predictive value of $86.4 \%$, and accuracy of 93.4 \%). Combined views ( FDG-PET/CT ) are better in evaluation.
\end{abstract}

Conclusion: FDG-PET is better in the detection of residual or recurrence in HCC which is locally managed by TACE. Keywords: HCC, FDG-PET/CT, Detection of recurrence or residual, TACE

\section{Background}

Hepatocellular carcinoma (HCC) or hepatoma is the most common primary malignancy of the liver. It ranks as the fifth most common tumor in the world and the third most common cause of cancer-related death [1]. However, in recent decades, HCC age-adjusted incidence rates have doubled. This is largely due to an increasing prevalence of ailments that predispose to hepatic cirrhosis, such as chronic viral hepatitis, obesity, and alcohol abuse [2].

Surgical treatment, including hepatic resection and liver transplantation, is considered as the most effective

Correspondence: mibraheim3333@gmail.com

Department of Diagnostic and Interventional Radiology, National liver Institute, Menoufia University, Al-Haram Al, Giza, Egypt treatment of HCC [3]. Patients with inoperable HCC were treated via interventional treatment [4].

Transarterial chemoembolization (TACE) works by blocking the primary feeder artery of the tumor by injection and concentration of chemotherapeutic agents at the tumor site [5].

Only $15-55 \%$ of patients have a partial response after TACE management. Therefore, evaluation of the accuracy of the therapy response and prognosis is important in determining if there will be a further session or not [6].

Positron emission tomography (PET) with 18F-2fluoro-2-deoxyglucose (18F-FDG) has become in recent years widely used for baseline staging and monitoring the treatment response in various tumors. 18F-FDG PET, which detects the glucose metabolic activity of tumors, provides useful information that 
cannot be obtained from other conventional imaging techniques $[7,8]$.

So 18F-FDG PET has become the most important imaging modality in the assessment of $\mathrm{HCC}$, before and after local management, as well as before and after liver transplantation, especially if combined with CT study. It gives additional information more than conventional modalities, so it will contribute to the clinical management of HCC recurrence [9].

\section{Methods}

\section{Patients}

- This was a diagnostic test accuracy study, performed on 46 patients (43 males (93.4\%) and 3 females $(6.6 \%))$.

- Their ages ranged between 37 and 61 years old with a median age of 50.5 years.

- They had pathologically proved HCC and managed locally by TACE. They came to the PET unit after 1 month from the last TACE.

- The patients were referred from the interventional unit to evaluate residual or recurrence post-TACE.

- The study was conducted from February 2018 to March 2019.

- All patients underwent the following:

- Thorough history taking

- Asking about previous allergy or reactions to contrast material

- Laboratory examination (serum creatinine and AFP level)

- Evaluation of the previous CECT

\section{Inclusion criteria}

- All patients with TACE for pathologically proved HCC.

\section{Exclusion criteria}

- Patients with previous allergy or reactions to contrast material

- Patients with no locoregional treatment of HCC

- Patients with locoregional treatment of HCC other than TACE

\section{The patients}

The patients included in the study already had previous TACE; we must know about its details (how many times, managed focal lesion criteria including site, size, and numbers).

\section{Patient preparation}

Before patient arrival

- A high-protein, low-carbohydrate diet is required $24 \mathrm{~h}$ before the scan

- Minimal physical activity within $24 \mathrm{~h}$ prior to the scan

- Good hydration with a full bladder; we may use diuretics

- Keeping patients in a warm environment to decrease brown fat uptake. We may use medications (beta blockers or diazepam). The dose of oral beta blocker (propranolol) is $20 \mathrm{mg}$ before FDG injection. And the dose of diazepam was $0.27 \mathrm{mg} / \mathrm{kg}$, maximum dose $10 \mathrm{mg}$

- Avoid muscular effort to decrease muscular uptake. Done by complete rest even from talking.

- Fasting at least $6 \mathrm{~h}$ before the study

- IV arm cannula is inserted with the removal of any metal objects that could produce artifact during the scan.

- Diabetic patients: patients were allowed to take early breakfast with oral anti-diabetic medications then fasting for $6 \mathrm{~h}$ or with their normal insulin dose and then fasting for $4 \mathrm{~h}$.

- Normal blood glucose level is important as tumor uptake decreased in hyperglycemic state. It must be $\leq 150 \mathrm{mg} / \mathrm{dl}$, and if more than $200 \mathrm{mg} / \mathrm{dl}$, PET/ $\mathrm{CT}$ is not performed. We may use insulin (2 to 5 units) to decrease the high blood glucose level, and blood glucose should be rechecked prior to FDG injection. A minimum time interval of $1 \mathrm{~h}$ is necessary between insulin administration and FDG injection to minimize undesirable effects which may be represented in increased FDG uptake in the heart, skeletal muscles, and liver which could affect image quality and therefore the ability to detect lesions

\section{PET and triphasic CT studies}

Done for all of these patients and evaluate the managed focal lesion and any newly developed one/s. All exams were performed using a Siemens Biograph 128 PET/CT with 128 slices CT.

- We gave $1 \mathrm{l}$ of negative oral contrast agent $1 \mathrm{~h}$ before examination. Then, the radioactive tracer is administrated as 10-20 mCi 18F-FDG with a dose of 0.1-0.4 mCi $/ \mathrm{kg}, 45-90 \mathrm{~min}$ before examination.

- Patients were asked to stay in a quiet dark room covered by warm blankets, not speaking, chewing, or reading, and then they were asked to void and taken to the scan room. 


\section{Protocol of PET/CT imaging}

- The examination was done from the base of the skull to the mid-thigh

- Low dose CT (without contrast) was done first

- PET protocol

- Immediately followed by emission scan imaging with multiple beds, at the same scan range in three-dimensional mode with $3 \mathrm{~min} / \mathrm{bed}$. Iterative algorithms \{TrueX+TOF (ultraHD-PET), 2 iterations, 21 subsets $\}$ were used for reconstruction of the PET images. PET axial field of view is 11.4 $\mathrm{cm} /$ bed position. Data were filtered (FWHM 4.0 $\mathrm{mm}$ ) and corrected for scatter.

- CECT protocol

- Then, contrast-enhanced triphasic CT (130 mAs, $120 \mathrm{kV}$, slice thickness $5 \mathrm{~mm}$, pitch 0.9 , and incremental reconstruction $2.5 \mathrm{~mm}$ ) was done after IV injection of $1.5-2 \mathrm{ml} / \mathrm{kg}$ of an iodinated contrast material (Optray 300) using automated injector (MedradStellant) with a flow rate of $4 \mathrm{ml} / \mathrm{s}$. The arterial phase on the liver was obtained after $20 \mathrm{~s}$ post-contrast injection, portovenous phase at $60 \mathrm{~s}$ delay starting from the skull base to the upper thighs (same as low dose and PET scan range), and delayed phase on the liver obtained at $300 \mathrm{~s}$ delay.

- A limited breath-hold technique was used to avoid motion-induced artifacts.

- Post-PET instructions

- The radioactive material remains in your body for about $12 \mathrm{~h}$, so we told patients to be limited in their contact with both pregnant women and infants during this time.

- Patients drunk plenty of fluids after the test to help flush the tracers out of their system. Generally, the tracers leave the body after 2 days.

\section{Image interpretation}

- All images were automatically fused on TrueDSemines software or transferred to Philips Intellispace portal and OSIRIX fusion workstation via the hospital network where FDG-PET and CT data sets were automatically fused.

- The PET and Triphasic CT images were interpreted and revised by two consultant radiologists with 5-10 years film reading, knowing previous patient's history of TACE (how many times, managed focal lesion criteria including site, size, and numbers)

\section{FDG-PET images}

- Visual analysis of axial, sagittal, and coronal reconstructed images was done first.

- The standard cutoff level of us depends on the uptake of the background of normal hepatic parenchyma which ranges from 2.5 to 4.5 in SUV, so we considered any area of increased FDG more than these values, areas of residual, or recurrence in managed HCC.

- Quantitative analysis to measure the degree of FDG uptake at detected lesions was done represented by maximum standardized uptake value (SUVmax)

- This was done by applying circular ROI with an average diameter of $2 \mathrm{~cm}$ over the most active part of HCC and extrahepatic metastasis.

\section{CECT images}

- Evaluated at axial, coronal, and sagittal reconstructed images

- Evaluate the TACE managed by focal lesion regarding size, site, unilobar or bilobar, lipidol retention, contrast enhancement, and its pattern in the three phases. Any new lesions

- Then, the correlation between FDG-PET uptake and tumor enhancement criteria in triphasic CT of the managed lesion was done.

The reference standard for us in accuracy determination is patients follow-up clinically, laboratory (mostly serum Alfa-fetoprotein level), and radiologically by deferent imaging modalities such as ultrasonography, CECT, dynamic MRI, or PET/CT. The most commonly used imaging modalities in follow up-beside serum Alfa fetoprotein level-were ultrasonography and CECT in

Table 1 Imaging features of the managed lesions

\begin{tabular}{llll}
\hline $\begin{array}{l}\text { Imaging } \\
\text { modality }\end{array}$ & Managed lesion criteria & $\begin{array}{l}\text { Number of } \\
\text { patients }\end{array}$ & $\begin{array}{l}\text { Percentage } \\
(\%)\end{array}$ \\
\hline PET & Increased tracer uptake & 38 cases & 82.6 \\
& No uptake (photopenic) & 8 cases & 17.4 \\
CECT & Contrast enhancement (arterial wash in and delayed wash out) & 26 cases & 56.5 \\
& Atypical contrast enhancement (marginal arterial enhancement with no washout or faint arterial & 6 cases & 13 \\
& enhancement) & 14 cases & 30.5 \\
\hline
\end{tabular}


Table 2 Correlation between positive and negative results in true, PET, and CECT study

\begin{tabular}{lllll}
\hline & Positive cases & Percentage (\%) & Negative cases & Percentage (\%) \\
\hline True reference cases & 34 & 74 & 12 & 26 \\
PET & 38 & 82.5 & 8 & 17.5 \\
CECT & 32 & 69.5 & 14 & 30.5 \\
\hline
\end{tabular}

38 cases, then dynamic MRI in 6 cases, and finally PET/ $\mathrm{CT}$ in only two cases.

\section{Statistical analysis}

The data collected were tabulated and analyzed by SPSS (Statistical Package for the Social Science Software) statistical package version 22 on IBM-compatible computer.

- We determined the sensitivity, specificity, positive predictive value, negative predictive value, and accuracy of FDG/PET in the detection of recurrence or new lesions compared to CECT, using data from the follow-up of patients (clinical, laboratory, and imaging) as reference for us in positivity or negativity of the obtained data.

\section{Results}

- The study was carried out on 46 patients (43 males (93.4\%) and 3 females (6.6\%)). And their ages ranged between 37 and 61 years old with a median age of 50.5 years.

- According to the follow-up data, which includes clinical picture, laboratory, and imaging methods, there were 34 patients who had local tumor recurrence or residual at the TACE-managed HCC.

- The evaluated managed $\mathrm{HCC}$ were more than $6 \mathrm{~cm}$ in max diameter in single lesions. Some of them are multiple and their sizes ranging from 4 to $7 \mathrm{~cm}$ in their average diameters. They were unipolar in all cases except two.

- PET study which is done after $1 \mathrm{~h}$ post-tracer administration, had increased pathological uptake at the TACE bed in 38 patients, while 8 patients had no pathological uptake at the TACE bed. Among the second group, we found that no patients had enhancement criteria of recurrence or residual tumor (Table 1).

- As for CECT, we found that 26 patients had typical pathological pattern of contrast enhancement at the TACE-managed HCC. This pattern means rapid arterial phase wash in and wash out in the portal and delayed phases. We found 6 patients had atypical pattern of enhancement in the form of either marginal arterial enhancement with no washout or faint arterial enhancement, while we found 14 patients had no

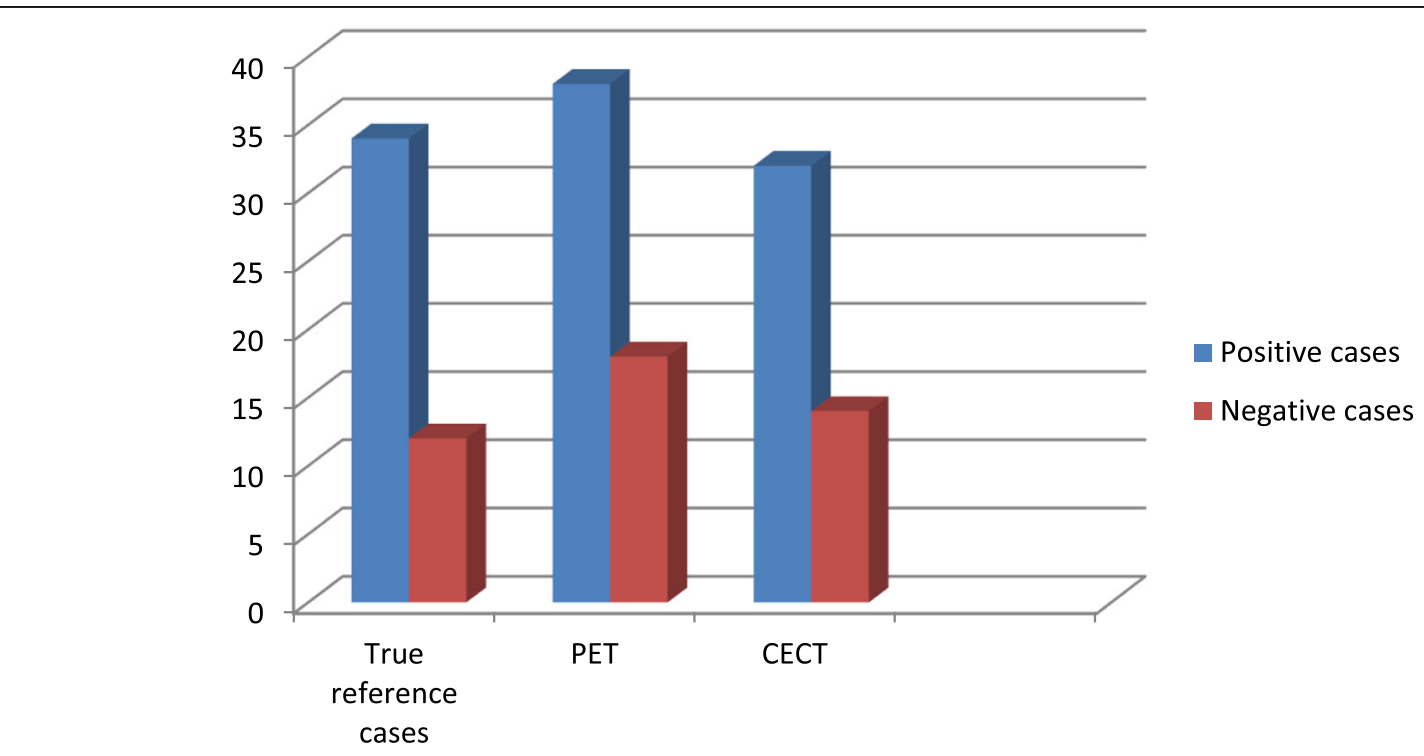

Fig. 1 Chart of correlation between positive and negative results in true, PET, and CECT study 
Table 3 Comparison of the statistical analysis of CECT and PET of the hepatic lesions managed by TACE

\begin{tabular}{llllll}
\hline Imaging modality & Sensitivity (\%) & Specificity (\%) & PPV (\%) & NPV (\%) & Accuracy (\%) \\
\hline CECT & 82 & 66.7 & 87.5 & 57.1 & 78.2 \\
PET & 100 & 66.7 & 89.5 & 100 & 91.3
\end{tabular}

enhancement criteria of recurrence. Among these patients with no recurrence or residual enhancement, there were 6 patients who showed FDG pathological uptake in PET scan, and 8 patients had no FDG pathological uptake (Table 1).

- From the previously collected data, we simply found that CECT showed true positive results in 28 cases, true negative in 8 cases, false negative in 6 cases, and false-positive results in 4 cases. However, PET showed true-positive results in 34 cases, truenegative in 8 cases, false-positive in 4 case, and no false-negative results.

- Table 2 shows the association between the true local residue/recurrence (which is detected in follow-up) and detection of local residue/recurrence by CECT and PET

The data seen in Table 2 could be presented in Fig. 1. Using data analysis, we could calculate the sensitivity, specificity, positive predictive value, negative predictive value, and accuracy for CECT and PET study results.

I found that CECT had a sensitivity of $82 \%$, specificity of $66.7 \%$, positive predictive value (PPV) of $87.5 \%$, negative predictive value (NPV) of $57.1 \%$, and accuracy of $78.2 \%$, while PET had sensitivity and specificity of $100 \%$ and $66.7 \%$ respectively. Positive predictive value (PPV) was $89.5 \%$, negative predictive value (NPV) was $100 \%$, and accuracy was $91.3 \%$ (Table 3 ).

\section{Cases}

Figures 2, 3, and 4 represent a sample of selected patients of our study, each figure for one case.

\section{Discussion}

Imaging plays an important role in the management of HCC, and the efficacy of treatment is usually monitored and assessed radiologically [10]. Generally, targeted therapy agents induce a reduction in tumor vascularization, provocation of necrotic area, and sometimes cavitation
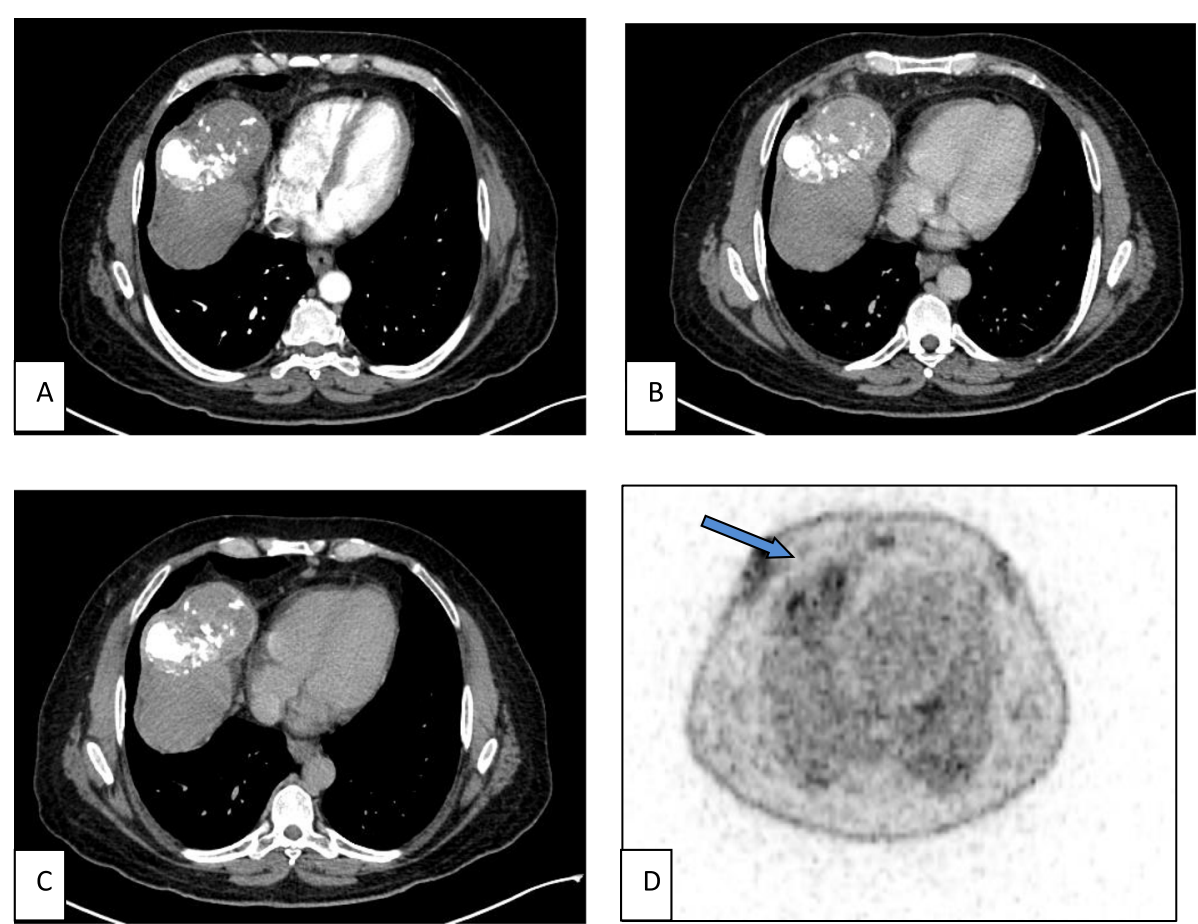

Fig. 2 Case (1). Male patient had done three sessions of TACE. CECT showed lipidole retention with no enhancement seen in arterial, portovenous, and delayed phases $(\mathbf{a}, \mathbf{b}, \mathbf{c})$. However, the PET scanning (d) of the same managed lesion showed significant FDG uptake in PET study (arrow), with SUV = 10. In follow-up, the managed lesion showed activity 

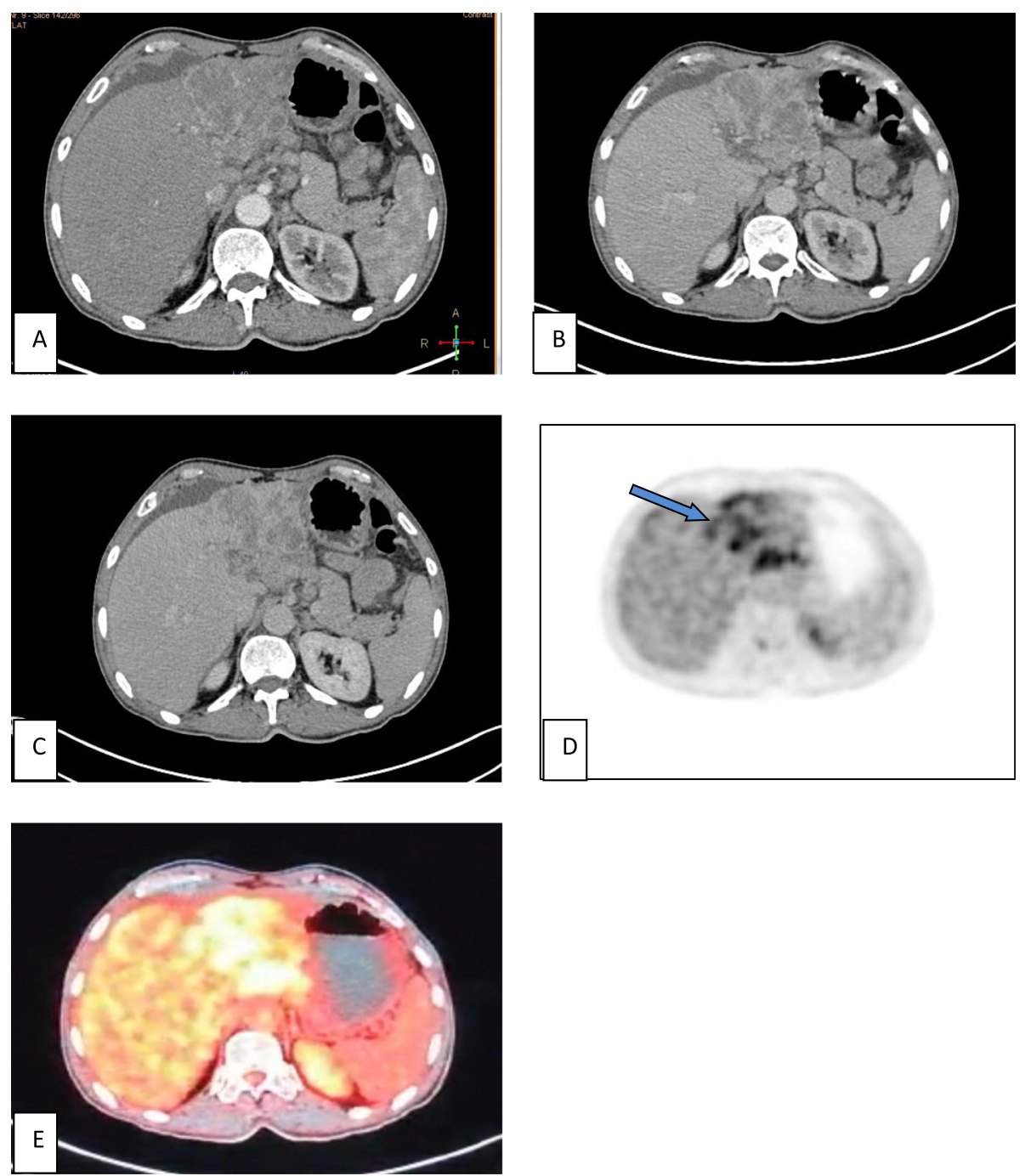

Fig. 3 Case (2). Male patient had done two sessions of TACE. CECT showed no lipidole retention with central breakdown and marginal enhancement seen in the arterial phase (a) and wash out in portovenous and delayed phases $(\mathbf{b}, \mathbf{c})$, more in delayed one. PET scanning (d). The same managed lesion showed marginal significant FDG uptake in PET study (arrow ) with (SUV = 13). In the follow-up, the managed lesion showed activity. Also, PET-CT image (e) shows significant FDG increased uptake compared to the liver parenchymal uptake

in solid tumors, and these features have been reported in various targeted therapies of HCC [11].

To evaluate the changes which occurred in the tumor after locoregional interventional procedures, we use many imaging modalities such as US, CECT, and dynamic MRI. However, lipidol deposits in TACE can make the evaluation more difficult in CECT, and dynamic MRI are the most commonly used tools to evaluate patients [12].

In HCC patients managed with TACE, contrastenhanced triphasic CT scan gives information about the tumoral size and its vascularity which may affect the recurrence or viability of the tumor. However, the main limitation of CT was the presence of hyperdense lipidol which may mask the viable intra-lesional tumor tissue [13].

FDG-PET depends on the assessment the metabolism of the tumor managed by TACE which is important in oncology. It has the advantage of examining the whole body as well, so we can assess the intraand extra-hepatic disease in single examination which is crucial for patients who planned for hepatic transplantation $[14,15]$.

Many studies had been done on the evaluation of the role of PET scan in evaluation of loco-regional ablation of the HCC. In this study, the efficiency of FDG-PET scan was compared to that of the CECT in the evaluation the local tumor residue/recurrence of HCC after TACE. 

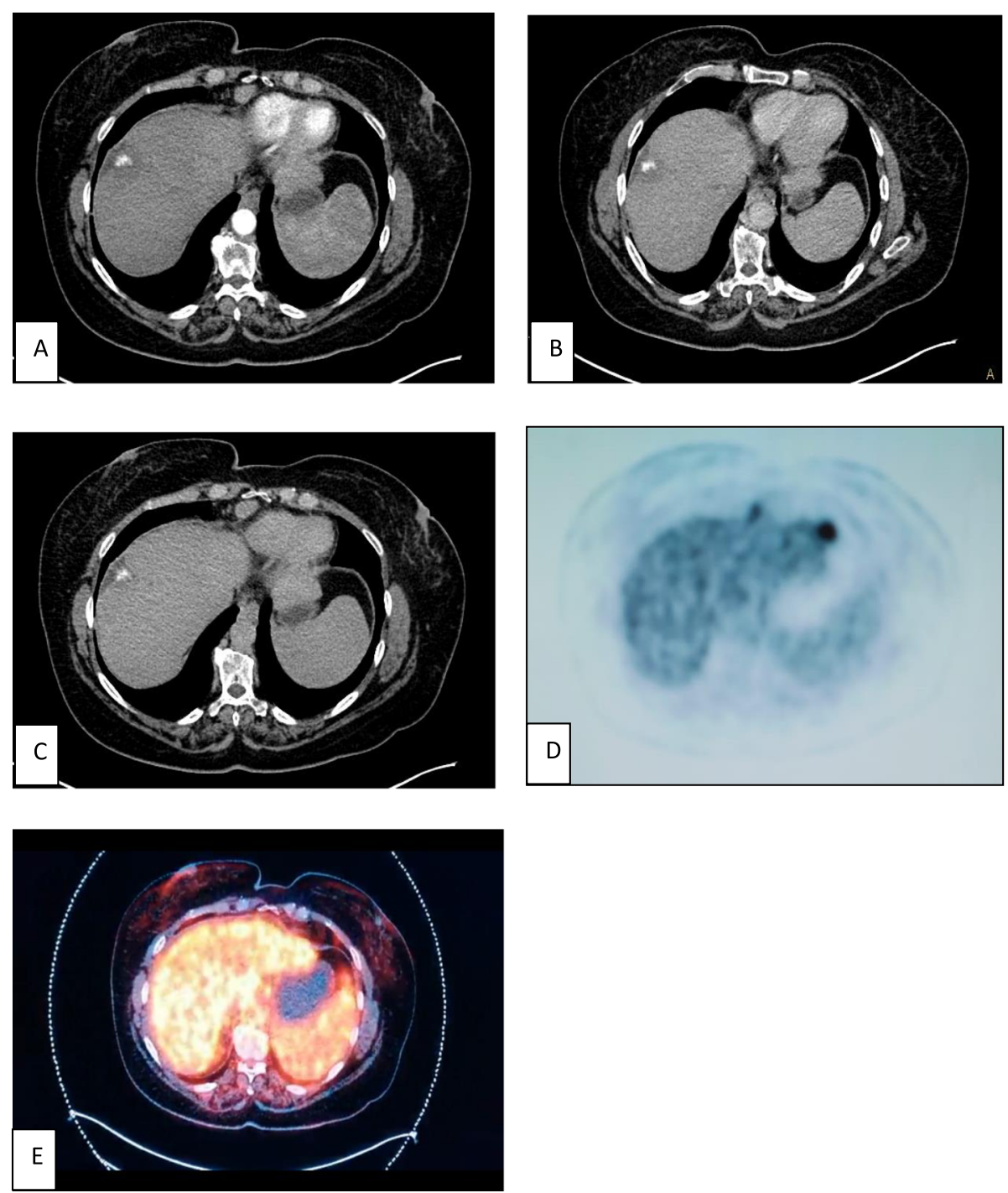

Fig. 4 Case (3). Female patient had done two sessions of TACE. CECT showed minimal lipidole retention with no enhancement seen in arterial, portovenous, and delayed phases ( $\mathbf{a}, \mathbf{b}, \mathbf{c})$. PET scanning (d) of the same managed lesion showed no significant FDG uptake in PET study (SUV = 4). In the follow-up, the managed lesion showed no activity. Also, PET-CT image (e) shows no increased uptake at the site of the managed lesion

It was found that the CECT had a sensitivity of $82 \%$, specificity of $66.7 \%$, positive predictive value (PPV) of $87.5 \%$, negative predictive value (NPV) of $57.1 \%$, and accuracy of $78.2 \%$. However, PET had sensitivity and specificity of $100 \%$ and $66.7 \%$, respectively. Positive predictive value (PPV) was $89.5 \%$, negative predictive value (NPV) was $100 \%$, and accuracy was $91.3 \%$.

Compared to Jinpeng et al. study [11] which studied the recurrence of HCC after (TACE) in 29 patients, they found the sensitivity of PET was $95.4 \%$ while the sensitivity of CECT was $63.8 \%$.

Also, Song et al. [16] study done in 2015 showed that F-FDG-PET/CT was found to be superior to CECT for the detection of viable tumor in patients with HCC after TACE.
Azab et al. [17] study concluded that FDG-PET/CT showed higher sensitivity and specificity than contrast $\mathrm{CT}$ in the evaluation of HCC after local therapy of HCC (which includes radiofrequency and TACE) whatever the degree of tumor vascularity.

Also, Wenhui et al. [18] study proved that 18F-FDG $\mathrm{PET} / \mathrm{CT}$ has diagnostic value in detecting viable $\mathrm{HCC}$ patients after TACE. Therefore, 18F-FDG PET/CT may provide valuable information that can be used in the treatment response evaluation and clinical decision-making process.

All the above-mentioned studies' results match with my results which showed that FDG PET was more accurate in the diagnosis of residual or recurrence HCC post-TACE than CECT which has an important role in deciding if the 
patient will take another session of TACE or not. But it still of limited role regarding cost, radiation exposure, more time of examination, and availability.

Also, using FDG-PET accuracy depends on the grade of biological activity of the tumor, meaning that the low biologically active tumor (low grade) may not be evaluated well by FDG-PET study.

The relatively small number of patients included in this study was considered as one of the weak points. Also, this study depends on the follow-up (imaging and laboratory) as the reference standard; however, histopathological correlation of the residue/recurrence is still more reliable as a reference standard.

\section{Summary}

In this study, it was found that 18 FDG-PET was better in the detection of recurrence or residual tumor in TACE-managed HCC lesions; this may be due to lipidole uptake which may mask some of the enhanced tissue activity or due to abnormal vascularity of the managed lesions. However, the combination of 18 FDGPET and CECT (PET/CT) surely gives better results.

\section{Abbreviations}

18-FDG: 18-Fluorodeoxyglucose; CECT: Contrast-enhanced computed tomography; HCC: Hepatocellular carcinoma; PET: Positron emission tomography; TACE: Transarterial chemoemoblization

\section{Acknowledgements}

We acknowledge firstly the patients who consented their data to be used, and the Radiology Department residents who help in collecting the data.

\section{Author's contributions}

MI Department of Diagnostic and Interventional Radiology, National Liver Institute-Menoufia University, gave the idea of the research, collected data, analyzed the data statistically, wrote the whole item of the paper, and was a contributor in writing the manuscript. Al-Haram Al-Giza. E mail (mibraheim3333@gmail.com).The author(s) read and approved the final manuscript.

\section{Funding}

No funding fees

\section{Availability of data and materials}

The datasets used and/or analyzed during the current study are available from the corresponding author on reasonable request. You could contact Dr. Manal Ibraheim.

\section{Ethics approval and consent to participate}

All study procedures were conducted in accordance with the Declaration of Helsinki and were approved by the ethical committee of the National Liver Instititute/Menoufia University, Egypt. The ethics committee reference number in the "Ethics approval and consent to participate" section is NLI IRB protocol number 00161. All patients included in this study gave us written consent to participate and all were adults, so no parental consent was taken.

\section{Consent for publication}

All patients included in this research gave written informed consent to publish the data contained within this study.
Received: 8 April 2020 Accepted: 23 June 2020

Published online: 27 July 2020

\section{References}

1. Altekruse SF, McGlynn KA, Reichman ME (2009) Hepatocellular carcinoma incidence, mortality, and survival trends in the United States from 1975 to 2005. J Clin Oncol 27(9):1485-1491

2. Montgomery J, Trebelev A, Bianco BA (2017) Imaging of hepatocellular carcinoma after transarterial locoregional therapy: a practical review and discussion of treatment response. J Am Osteopath Coll Radiol 6(3):5-12

3. Mazzaferro V, Regalia E, Doci R, Andreola S, Pulvirenti A, Bozzetti F et al (1996) Liver transplantation for the treatment of small hepatocellular carcinomas in patients with cirrhosis. N Engl J Med 334:693-699

4. Hayanon K, Lee S, And Sahani DV (2015) Imaging for assessment of treatment response in hepatocellular carcinoma: current update. Indian J Radiol Imaging 25(2):121-128

5. De Baere T, Arai, Lencioni R (2016) Treatment of liver tumors with Lipiodol TACE: technical recommendations from experts opinion. Cardiovasc Intervent Radiol 39:334-343

6. EASL-EORTC (2011) Clinical practice guidelines: management of hepatocellular carcinoma. J Hepatol 2012(56):908-943

7. Higashi T, Hatano E, Ikai I, Nishii R, Nakamoto Y, Ishizu K, Suga T, Kawashima H, Togashi K, Seo S, Kitamura K, Takada Y, Uemoto S (2010) FDG PET as a prognostic predictor in the early post-therapeutic evaluation for unresectable hepatocellular carcinoma. Eur J Nucl Med Mol Imaging 37: 468-482

8. Ahn SG, Kim SH, Jeon TJ, Cho HJ, Choi SB, Yun MJ, Lee JD, Kim KS (2011) The role of preoperative [18F]fluorodeoxyglucose positron emission tomography in predicting early recurrence after curative resection of hepatocellular carcinomas. J Gastrointest Surg 15:2044-2052

9. Kim YK, Lee KW, Cho SY (2010) Usefulness ${ }^{18}$ F-FDG positron emission tomography/computed tomography for detecting recurrence of hepatocellular carcinoma in posttransplant patients. Liver Transpl 16:767772

10. Hennedige T, Venkatesh SK (2013) Imaging of hepatocellular carcinoma: diagnosis, staging and treatment monitoring. Cancer Imaging 12:530-547

11. Jinpeng $L$, Wenbo S, Jinlong S (2013) The therapeutic effect of transcatheter arterial thromboembolization of hepatocellular carcinoma as for residual viable tumors related to lipiodol density areas detected by 18F-FDG PET/CT and CT. Hell J Nucl Med 16(1):64-65

12. European Association for the Study of the Liver (2018) Clinical practice guidelines: management of hepatocellular carcinoma. J Hepatol 69(1):182236 pmid:29628281

13. Dioguardi Burgio M, Ronot M, Bruno O (2016) Correlation of tumor response on computed tomography with pathological necrosis in hepatocellular carcinoma treated by chemoembolization before liver transplantation. Liver Transpl 2016(22):1491-1500

14. Risse JH, Pauleit D, Bender H (2009) 18F-FDG-PET and histopathology in 131 llipiodol treatment for primary liver cancer. Cancer Biother Radiopharm 24:445-452

15. Paudyal B, Oriuchi N, Paudyal P et al (2007) Early diagnosis of recurrent hepatocellular carcinoma with 18F-FDG PET after radiofrequency ablation therapy. Dep Diag Radiol Nucl Med Gunma Univ Grad School Med Oncol Rep 18:1469-1473

16. Song HJ, Cheng JY, Hu SL (2015) Value of 18F-FDG PET/CT in detecting viable tumour and predicting prognosis of hepatocellular carcinoma after TACE. Clin Radiol 70(2):128-137

17. Azab OA, Ali M, El-Refaei SM (2016) Role of 18-F FDG-PET/CT in the detection of local tumor residue/recurrence in hepatocellular carcinoma (HCC) post hepatic therapeutic intervention. Med J Cairo Univ 84(1):991-998

18. Wenhui MA, Jia J, Shengjun W (2014) The prognostic value of 18F-FDG PET/ CT for hepatocellular carcinoma treated with transarterial chemoembolization (TACE). Theranostics 4(7)

\section{Publisher's Note}

Springer Nature remains neutral with regard to jurisdictional claims in published maps and institutional affiliations. 